\title{
Performance Analysis of Single Quantum Dots and Couple Quantum Dots at Intermediate Band Solar Cells
}

\author{
Sajad Heshmati ${ }^{1}$, Saeed Golmohammadi ${ }^{2}$ \\ ${ }^{1}$ Department of Electrical Engineering,Buin Zahra Branch, Islamic Azad University, Buin Zahra, Qazvin, Iran \\ ${ }^{2}$ Nanophotonics Group, School of Engineering-Emerging Technologies, University of Tabriz, Tabriz
} 5166614761, Iran

\begin{abstract}
In this paper, the performance of the single quantum dots and couple quantum dots at intermediate band solar cells are investigated. Simulation results show that by increasing the distance between quantum dot overlap between the wave functions of the quantum dots is reduced and the intermediate bandwidth decreases. We found that an intermediate band has an inverse relation with efficiency.

Keywords: Barrier width, Efficiency, Quantum dot intermediate band solar cell (QD-IBSCs) ' Quantum-dot width · Efficiency.
\end{abstract}

\section{I . INTRODUCTION}

Quantum dot intermediate band solar cells (QD-IBSCs) is the newest type of solar cells for achieving the maximum attainable efficiency. Realization of low-cost photovoltaic power sources is subject to development of low cost and efficient solar cells. The performance of the intermediate band solar cell (IBSC) depends on the electrical and optical properties of the intermediate band (IB) material. The intermediate band (IB) solar cells utilize more of the incoming photons through using an intermediate band placed in the band gap between the conduction and valence band. The band gap of the semiconductor is divided into two sub-bands gaps. [1] These are characterized by an electronic band that is located between the conduction band (CB) and the valence band (VB) of a conventional semiconductor band gap. The IBSC has been proposed by using quantum dot (QD) technology [2], [8].

The structure of the QDs plays an important role to achieve maximum efficiency. The energy conversion efficiency is a fundamental parameter in the photovoltaic solar cell technology [4]. It is defined as, (1)

$$
\eta=F F \cdot V_{O C} \cdot J_{S C} / P_{\text {in }}
$$

Where FF is the fill factor $V_{\text {os }}$ is the open circuit voltager $J_{\text {sc }}$ is the short-circuit current density، and $P_{\text {in }}$ is the incident power per unit area [4]. A schematic of the IBSC is shown in fig. 1.

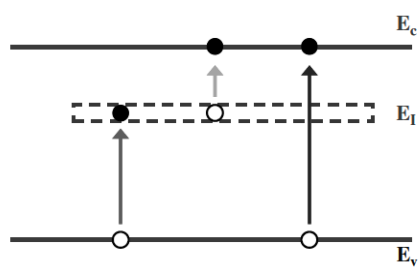

Figure1. IBSC [12]

The ideal performance of the solar cells the bandgaps separating the IB from the $\mathrm{CB}$ and separating the IB from the $\mathrm{VB}$ are in the range 1.24EV, and $0.71 \mathrm{EV}$, respectively [3], [5]. These bandgaps are shown in fig. 2. These gaps can be exchanged, for example the IB could be closed to the VB respect to the CB. On the other hand، the IB must be partially filled with electrons، so that empty states has been existed for receiving the electrons pumped from the VB and also electrons-filled states to provide for the electrons pumped to the CB [7].

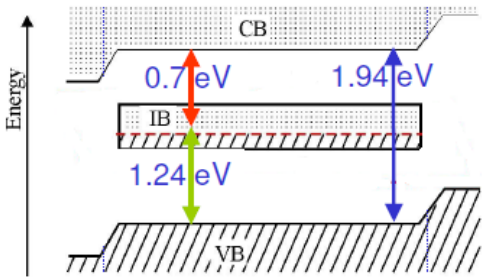

Figure2. IBSC with optimum bandgap 
A semiconductor with single bandgap only absorbs photons with energies above the bandgap threshold. Therefore ' only these photons contribute in the produced photocurrent. But when there is a half-filled IB، two photons with energies below the bandgap threshold can pump an electron from the VB to the CB. A first photon with energy greater than $\mathrm{E}_{l}$ will pump an electron from the VB to the IB that has empty states for receiving the electron and a next photon with energy greater than $E_{h}$ pump an electron from the IB that has electron-filled states to the $\mathrm{CB}$ [6]. In this method, photocurrent is greater than the current that can be produced by semiconductors with single bandgap [3], [5], [7]. In the radiative limit the IBSC shows a limit efficiency of $63.2 \%[3,5]$ ، significantly higher than the single gap solar cell efficiency $(40.7 \%)$ and two-junction solar cell efficiency $(55.4 \%)$, operated at their radiative limit. However in this paper the relationship between various parameters of the QDs such as CB offset، QDs width and QDs barrier width for achieving the maximum efficiency is investigated. This article is in four parts. In the first part (II) of the QD-IBSCs design rules have been explained. In the second part of the analysis of single and coupled quantum dots have been studied and their relationship with IB is investigated. In the third part of the quantum dot conduction band energy using of effective mass approach is calculated and two material systems using of kronig-penny model have been investigated. The final section concludes shown.

\section{Design Rules for QD-IBSC}

Design rules for selecting the QD-IBSC materials triad (QD/Barrier/Substrate) is defined as [3], [9], [13]

A. The barrier material must have a bandgap $\mathrm{E}_{\mathrm{GB}}$ in the interval $[1.43 \mathrm{eV}, 2.56 \mathrm{eV}]$.

B. The valence band offset must be negligible.

C. Material with direct bandgap must be used because they have a larger absorption coefficient

D. The offset between the $\mathrm{CB}$ edges $\mathrm{E}_{\mathrm{CB}}$ must be greater than $0.48 \times \mathrm{E}_{\mathrm{CB}}-0.22$.

E. The mean distance between the self-assembled QDs should be such that the overlap between the intermediate band and $\mathrm{CB}$ and $\mathrm{VB}$ is prohibited.

\section{Analysis of the Single DQ and Coupled QD and their relationship with IB}

In order to study the performance of the QD-IBSCs, two cases are investigated:

A. When the quantum dots are located at great distances from each other and do not overlap with each other.

B. When the QDs are closed to each other their wave functions overlap with each other to form an intermediate band [8].

The wave functions of a Single QDs and a set of coupled QDs are shown in fig. 3.

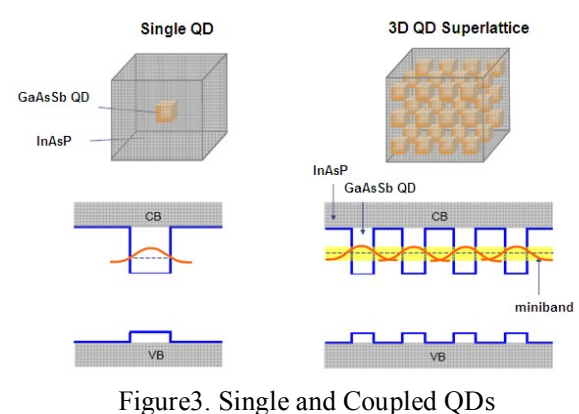

IV. Calculation of QD-CB energy using of effective mass approach

The conduction band energy of an idealized version of a three-dimensional silicon QD superlattice can be calculated using an effective mass approach [10]. Silicon QD superlattice with a regularly spaced array of equally sized cubic dots in a dielectric matrix is shown in fig. 4 [10].

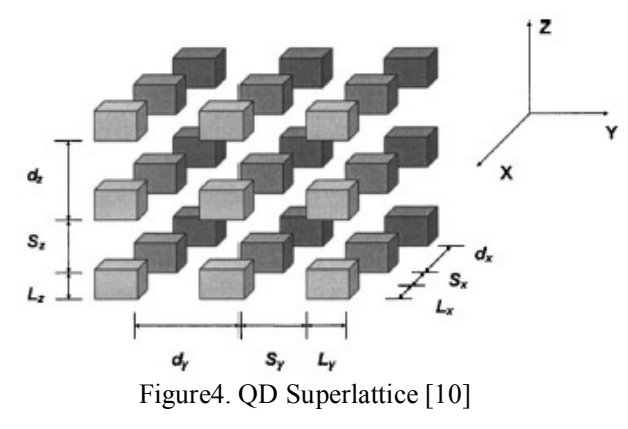


In this approach، the motion of a carrier in the material system is defined by the effective mass equation which is defined as, (2) [10]

$$
\frac{\hbar^{2}}{2} \nabla\left[\frac{1}{m^{*}(r)} \nabla \varphi(r)\right]+[E-V(r)] \varphi(r)=0
$$

Where $\mathrm{m}^{*}$ (r) is the effective mass tansor، $\mathrm{E}$ is the total energy and $\varphi(r)$ is the envelope function of the electron. $\mathrm{V}(\mathrm{r})$ is the microscopic potential seen by the electron which is considered the sum of three independent periodic functions. It is defined as, (3)

$$
V(r)=V_{x}(r)+V_{y}(r)+V_{z}(r)
$$

Consequently, the three dimensional effective mass equation is separable for the case of isotropic effective mass and is reduced to three one-dimensional quantum well superlattice equations [11]. As a result, the solution of Eq. (2) Can be expressed in terms of solutions of the simple one-dimensional kronig-penny model [11]. In the case of the isotropic effective mass the following equations are defined as, (4)

$$
\begin{aligned}
& \cos \left(q_{i} d_{i}\right)=\cos \left(k_{i}^{D} L_{i}\right) \cos \left(k_{i}^{B} S_{i}\right)-\frac{1}{2} \\
& \left(\frac{k_{i}^{B} m_{D i}^{*}}{k_{i}^{D} m_{B i}^{*}}+\frac{k_{i}^{D} m_{B i}^{*}}{k_{i}^{B} m_{D i}^{*}}\right) \times \sin \left(k_{i}^{D} L_{i}\right) \sin \left(k_{i}^{B} S_{i}\right) f f E_{i} \geq V_{0} \\
& \cos \left(q_{i} d_{i}\right)=\cos \left(k_{i}^{D} L_{i}\right) \cosh \left(k_{i}^{B} S_{i}\right)-\frac{1}{2} \\
& \left(\frac{k_{i}^{B} m_{D i}^{*}}{k_{i}^{D} m_{B i}^{*}}+\frac{k_{i}^{D} m_{B i}^{*}}{k_{i}^{B} m_{D i}^{*}}\right) \times \sin \left(k_{i}^{D} L_{i}\right) \\
& \sinh \left(k_{i}^{B} S_{i}\right) i f 0 \prec E_{i} \prec V_{0}
\end{aligned}
$$

In, (4) and (5) $k_{i}^{B}=\sqrt{2 m_{B i}^{*}\left|E_{i}-V_{0}\right|} / \hbar ، m_{B i}^{*}$ is the effective mass in the dielectric matrix and $k_{i}^{D}=\sqrt{2 m_{D i}^{*}\left|E_{i}\right|} / \hbar \cdot m_{D i}^{*}$ is the effective mass of the QD، $q_{i}$ is the wave vector component and $i$ is the coordinate direction [11]. The energy dispersion relation is then obtained from, (6)

$$
E(q)=E_{x}(q)+E_{y}(q)+E_{z}(q)
$$

By using of kronig-penny model [11,12], The following analysis for different values of the barrier width and the width of the quantum dots is investigated. In the simulations، the two material systems have been analyzed:

A. In the following analysis $\mathrm{GaAs}_{0.98} \mathrm{Sb}_{0.02}$ as Barrier material and $\operatorname{InAs}_{0.49} \mathrm{P}_{0.51}$ as QD material is considered. AlAs is used as a substrate. Bandgap of the barrier material is 1.48EV and bandgap of the QDs is 0.91EV. The effective mass of the barrier material is $0.066 \mathrm{~m}_{0}$ and effective mass of the QDs is $0.039 \mathrm{~m}_{0}\left(\mathrm{~m}_{0}\right.$ is the rest mass of the electron). In this material system there is an energy difference of $\sim 0.57 \mathrm{EV}$ between the conduction band energy of the $\mathrm{GaAs}_{0.98} \mathrm{Sb}_{0.02}$ and conduction band energy of the $\operatorname{InAs}_{0.49} \mathrm{P}_{0.51}[9]$.

Diagram of the energy dispersion versus quantum dot electron wavefunction is shown in fig 5, 6, 7 .

Bandstructure Dispersion

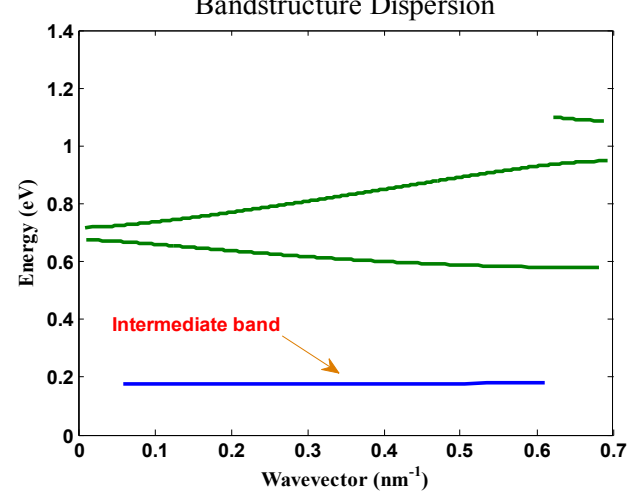

Fig.5: diagram of the energy dispersion versus quantum dot electron wavefunction to dot width (4nm) and barrier width (6nm) and intermediate bandwidth is very thin 


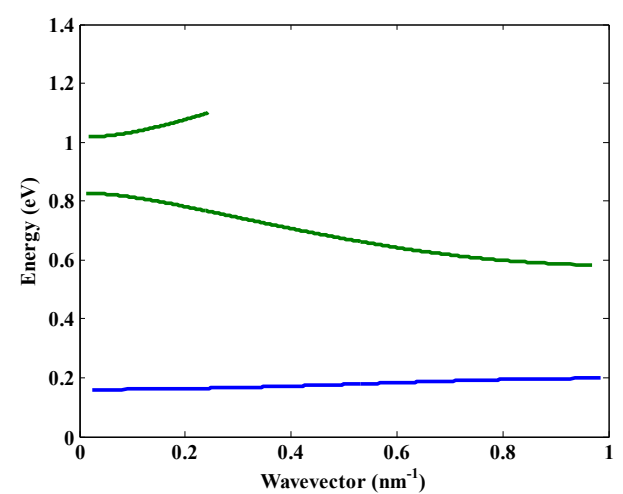

Fig.6: diagram of the energy dispersion versus quantum dot electron wavefunction to dot width (4nm) ، barrier width ( $3 \mathrm{~nm})$ and intermediate bandwidth $(30 \mathrm{meV})$

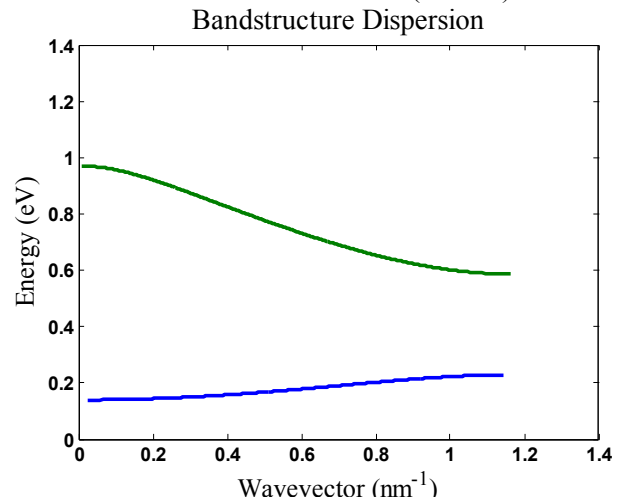

Fig.7: diagram of the energy dispersion versus quantum dot electron wavefunction to dot width (4nm), barrier width (2nm) and intermediate bandwidth $(90 \mathrm{meV})$

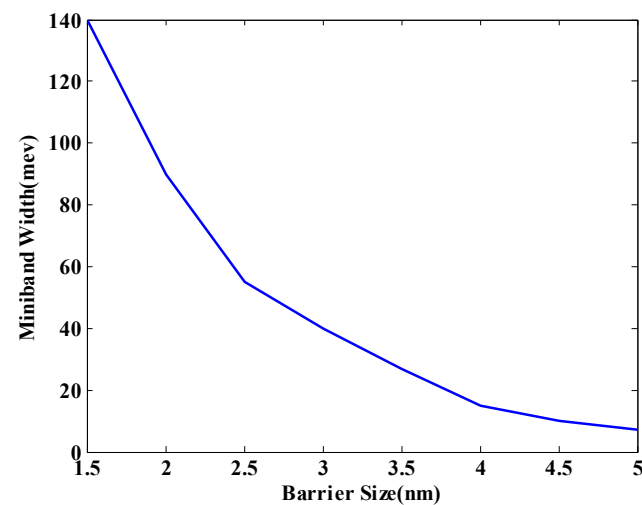

Fig8: diagram of the intermediate bandwidth versus barrier width

Table1: The values of the intermediate bandwidth and efficiency with different parameters of QDs

\begin{tabular}{lccc}
\hline Efiiciency(\%) & $\begin{array}{c}\text { Intermediate } \\
\text { band } \\
\text { width(meV) }\end{array}$ & $\begin{array}{c}\text { Barrier } \\
\text { width(nm) }\end{array}$ & $\begin{array}{c}\text { Quantum dot } \\
\text { width(nm) }\end{array}$ \\
\hline 54.51 & 3 & 6 & 4 \\
54.42 & 7 & 5 & 4 \\
54.15 & 17 & 4 & 4 \\
\hline 53.64 & 38 & 3 & 4 \\
52.52 & 90 & 2 & 4 \\
\hline
\end{tabular}

As shown in fig 5, QDs are located far from each other. In this structure, The first band (electron wave function) is considered as an IB. Since the QDs are widely separated, they do not overlap with other parts of the wave functions of QDs. As a result, the intermediate bandwidth is reduced. In fig 6 and 7, QDs are placed in close distance and therefore the QDs wavefunction has overlap with each other and energy band (electron wavefunctions) is wider. The result is the formation of an IB. In fig 8, Intermediate bandwidth decreases with increasing barrier width. In table 1 efficiency has an inverse relationship to intermediate bandwidth .( The calculations under air mass AM1.5G and solar intensity 1000 sun is performed) 
B. In the following analysis $\mathrm{GaAs}_{0.98} \mathrm{Sb}_{0.02}$ as Barrier material and $\mathrm{InP}_{0.82} \mathrm{Sb}_{0.18}$ as QD material is considered. AlAs is used as a substrate. Bandgap of the barrier material is $1.48 \mathrm{EV}$ and band gap of the QDs is $0.92 \mathrm{eV}$. The effective mass of the barrier material is $0.066 \mathrm{~m}_{0}$ and effective mass of the QDs is $0.042 \mathrm{~m}_{0}\left(\mathrm{~m}_{0}\right.$ is the rest mass of the electron). In this material system ' there is an energy difference of $\sim 0.56 \mathrm{EV}$ between the conduction band energy of the $\mathrm{GaAs}_{0.98} \mathrm{Sb}_{0.02}$ and conduction band energy of the $\mathrm{InP}_{0.82} \mathrm{Sb}_{0.18}$ [7].

Dispersion diagram of the band structure (energy versus wave vector) with QDs fixed width and different values of the barrier width is shown in fig 9, 10, 11. The results shown in table 2 that with increasing barrier width, Intermediate bandwidth is reduced and vice versa.

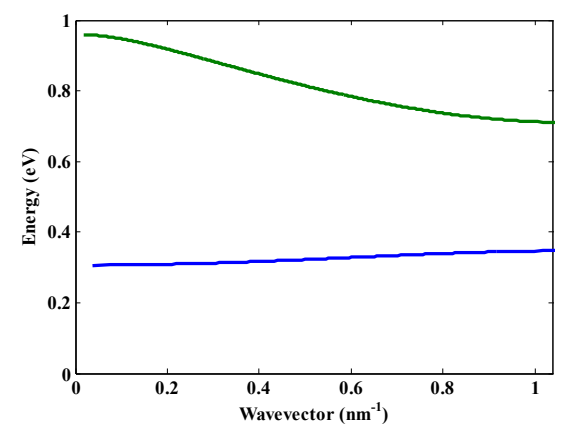

Fig 9: dot width (2nm), barrier width $(4.5 \mathrm{~nm})$ and intermediate bandwidth (70meV)

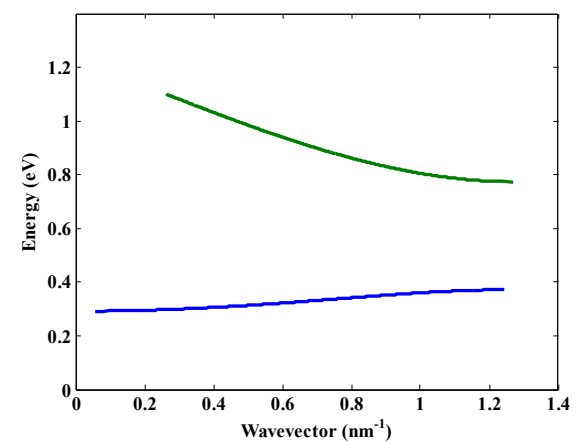

Fig 10:dot width (2nm), barrier width $(3.5 \mathrm{~nm})$ and intermediate bandwidth $(110 \mathrm{meV})$

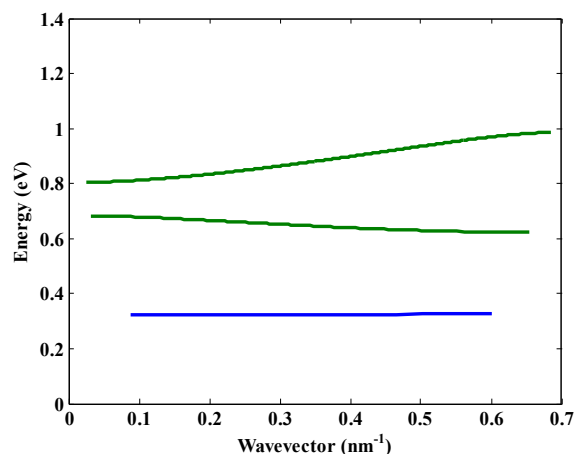

Fig 11: dot width $(2 \mathrm{~nm})$, barrier width $(8 \mathrm{~nm})$ and intermediate bandwidth $(25 \mathrm{meV})$

Table 2: The values of the intermediate bandwidth and efficiency with different parameters of quantum dot

\begin{tabular}{ccc}
\hline $\begin{array}{r}\text { Intermediate band } \\
\text { width(meV) }\end{array}$ & Barrier width(nm) & $\begin{array}{r}\text { Quantum dot } \\
\text { width(nm) }\end{array}$ \\
\hline 110 & 3.5 & 2 \\
90 & 4 & 2 \\
70 & 4.5 & 2 \\
55 & 5 & 2 \\
30 & 7 & 2 \\
25 & 8 & 2 \\
\hline
\end{tabular}

In Table 2, the values of the intermediate bandwidth with a width of $2 \mathrm{~nm}$ QDs has been investigated and in table 3, the values of the intermediate bandwidth with a width of $3 \mathrm{~nm}$ QDs has been investigated. Dispersion diagram of the band structure (energy versus wavevector) is shown in fig 12, 13,14. In Tables 1,2 , 3, different 
amounts of intermediate bandwidth with the constant quantum dots and different values of the barrier width is calculated. The results in the tables show that intermediate bandwidth decreases with increasing barrier width and vice versa and also in Table 3 is shown that efficiency decreases with increasing the width of the middle band and also efficiency by reducing the width of the middle band increases(The calculations under air mass AM1.5G and solar intensity 1000 sun is performed).

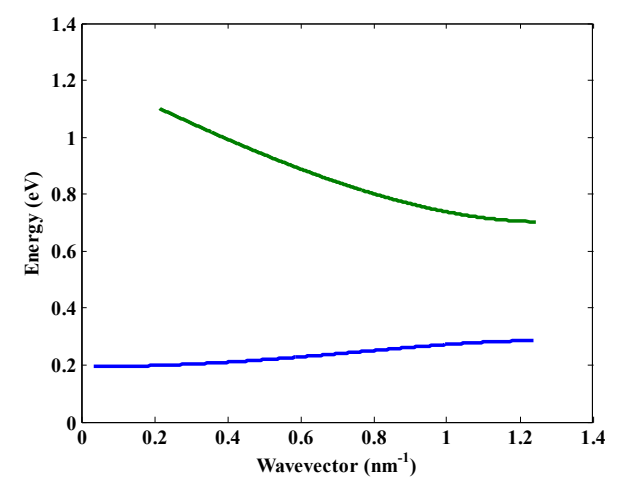

Fig 12: dot width $(3 \mathrm{~nm})$, barrier width $(2.5 \mathrm{~nm})$ and intermediate bandwidth $(93 \mathrm{meV})$

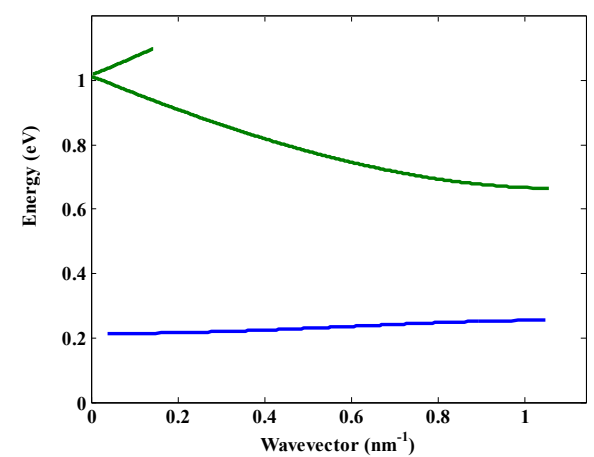

Fig 13: dot width (3nm), barrier width $(3.5 \mathrm{~nm})$ and intermediate bandwidth $(43 \mathrm{meV})$

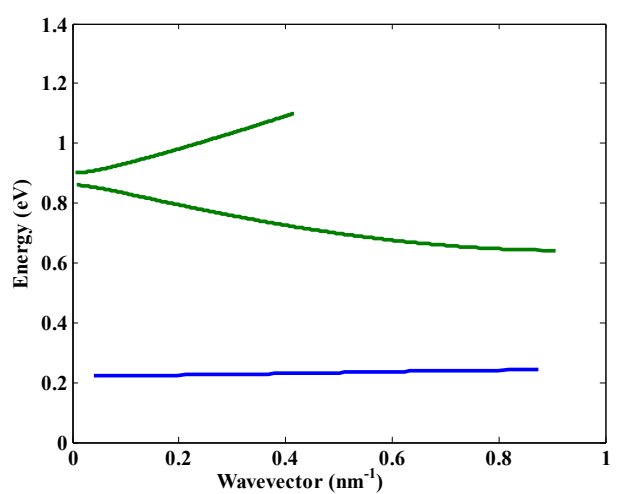

Fig 14: dot width (3nm), barrier width $(4.5 \mathrm{~nm})$ and intermediate bandwidth $(20 \mathrm{meV})$

Table 3: The values of the intermediate bandwidth and efficiency with different parameters of quantum dot

\begin{tabular}{cccc}
\hline Efiiciency(\%) & $\begin{array}{c}\text { Intermediate } \\
\text { band } \\
\text { width(meV) }\end{array}$ & $\begin{array}{c}\text { Barrier } \\
\text { width(nm) }\end{array}$ & $\begin{array}{c}\text { Quantum dot } \\
\text { width(nm) }\end{array}$ \\
\hline 51.18 & 93 & 2.5 & 3 \\
51.66 & 63 & 3 & 3 \\
52.10 & 43 & 3.5 & 3 \\
52.28 & 29 & 4 & 3 \\
52.41 & 20 & 4.5 & 3 \\
\hline
\end{tabular}




\section{Conclusion}

Analysis results in two material systems above shows that in QD-IBSCs, intermediate bandwidth by increasing the width of the barrier, is reduced and intermediate bandwidth by reducing the width of the barrier is increased. The intermediate band has an inverse relationship with the efficiency. Finally increase or decrease the intermediate bandwidth is effective in increasing and reducing efficiency. We found that an intermediate band has an inverse relation with efficiency.

\section{References}

[1] P. F. Hamedan, A. Taghini, F. Yazdi, Z. Mirmoeini, "Study the Effect of Varying the Thickness of Intermediate Band on the Intermediate Band Solar Cells Efficiency” Energy Procedia, 14, 1496-1502, (2012).

[2] M. A. Green, "Potential for low dimensional structures in photovoltaics,"Mater. Sci. Eng., vol. 74, pp. 118-124, 2000.

[3] A. Luque and A.Martí, "Increasing the efficiency of ideal solar cells by photon induced transitions at intermediate levels," Phys. Rev. Lett, 78 (26) 5014-5017, 1997.

[4] Q. Shao and A. Balandin. "Intermedite-band solar cells based on quantum dot supracrystals" appl. Phys. Lett. 91, 163503 (2007).

[5] A. Luque and A. Martí, "A metallic intermediate band high efficiency solar cell," Prog.Photovoltaics, Res. Applicat, vol. 9, no. 2, pp. 73-86, 2001 .

[6] N. Lopez, A. Marti and A. Luque, "Experimental Analysis of the Operation of Quantum Dot Intermediate Band Solar Cells," Journal of Solar Energy Engineering, vol. 129, no. 2, pp. 319_321, 2007.

[7] A. Martí et al, "Partial Filling of a Quantum Dot Intermediate Band for Solar Cells," IEEE Trans. on Elec. Dev.48, pp. 2394-2399, 2001 .

[8] A. Mart, L Cuadra and A. Luque, "Quantum dot intermediate band solar cell," in Proceedings of the 28th IEEE Photovoltaics Specialists Conference, pp. 940-943, IEEE, New York, 2000.

[9] M. Y. Levy, C. Honsberg, A. Marti, and A. Luque, "Quantum Dot Intermediate Band Solar Cell Material Systems With Negligible Valence Band Offsets," Proceedings of the 31st IEEE Photovoltaic Specialists Conference, IEEE, New Jersey, pp.90-93, 2005.

[10] Chu. Wei. Jiang and M. A. Green,"Silicon quantum dot superlattices: Modeling of energy bands, densities of states, and mobilities for silicon tandem solar cell applications," J.Appl.Phys.99, 114902, 2006.

[11] O. Lazarenkova and A. Balandin,"Miniband formation in a quantum dot crystal," J. Appl. Phys. 89, 5509, 2001.

[12] R. Aguinaldo,"Modeling Solutions and Simulations for Advanced III-V Photovoltaics Based on Nanostructure," Rochester Institute of Technology, 2008

[13] S. Jenks and R. Gilmore, "Quantum dot solar cell:Materials that produce two intermediate bands," Journal of Renewable and Sustainable Energy, vol .2, pp. 013111, 2010. 